lismus und ausländischen Monopolen, wobei der nationalen Industriebourgeoisie die Rolle eines untergeordneten Juniorpartners zukommt.

Die sozialen Maßnahmen der Militärs zielen ab auf die Stärkung der schmalen Mittelschicht, der sie selbst mehrheitlich entstammen. Während für die Masse der Arbeiterschaft und der Landbevölkerung die Löhne gesunken und die Arbeitsplätze knapper geworden sind, gibt die punktuell durchgeführte Landreform und das neue Betriebsverfassungsrecht einer kleinen Gruppe von Arbeitnehmern die Chance eines Aufstiegs zu einer Arbeiteraristokratie. Gewerkschaftliche Organisationen werden zunehmend reprimiert und ersetzt durch "Gilden", in denen alle "Arbeiter" eines Betriebs bis hinauf zum ersten Arbeiter: dem Unternehmer, in konfliktfreier Harmonie vereinigt sein und damit den „scheinbar unausweichlichen“ Gegensatz von Arbeit und Kapital überwinden sollen. Die in diesen "Gilden" herrschende „Mitbestimmung“ und „Gewinnbeteiligung“ bevorzugen auf jede erdenkliche Weise die Unternehmensspitze und erweisen sich somit als pure Augenwischerei.

Quijano geht nicht auf die oftmals diskutierte Frage ein, ob ein Reformregime wie das peruanische spätere grundlegendere Reformen vorbereiten helfe oder ihnen im Gegenteil den Weg verstelle. Der hohe Abstraktionsgrad der Arbeit birgt die Gefahr, die Widersprüchlichkeiten und Unklarheiten einer „erratischen Politik“ hinwegzuabstrahieren und eine trügerische Klarheit entstehen zu lassen. Dies kann jedoch nicht als Nachteil betrachtet werden bei einer Arbeit, die sich ausdrücklich zum Ziel setzt, in knappster Form versuchsweise Hypothesen über die wesentlichen Merkmale des neuen Regimes zu formulieren.

Der vorliegende Band ist ein Beispiel für die enormen Fortschritte, die der Sozialwissenschaft bei der Analyse lateinamerikanischer Probleme in den letzten Jahren gelungen ist - unter Führung der Lateinamerikaner selbst.

Tilman Tönnies Evers

Jean J. A. Salmon

La Reconnaissance d'État

Quatre Cas: Mandchoukouo, Katanga, Biafra, Rhodésie du Sud

Verlag Armand Colin, Paris 1971, 287 S.

Salmon bietet keine theoretische oder historische Abhandlung über das Problem der Anerkennung von Staaten, sondern ein didaktisches Instrument, das für Studenten wie Lehrer des Völkerrechts, der internationalen Beziehungen und verwandter Disziplinen von großem Nutzen sein kann. Das kleine Werk erfüllt diesen Zweck in ausgezeichneter Weise, und man kann ihm nur weite Verbreitung wünschen. Es gliedert sich in zwei ungleiche Teile. Der erste, absichtlich klein gehaltene enthält eine knappe, aber durchaus informative Einführung in die theoretische Problematik der Anerkennung, ihre nach Objekt und Form verschiedenen Spielarten, die Grenzen der Anerkennungsfreiheit (Salmon verneint die Rechtspflicht zur Anerkennung und zieht wie üblich die Grenze der Anerkennungsfreiheit bei der Intervention, setzt sie aber auch mit dem Selbstbestimmungsrecht in Beziehung) und die Situation des nicht anerkannten Staates (die Literatur über sie erwähnt leider nicht die wichtige Arbeit von J. A. Frowein hierzu). Wenn auch kurz, behandelt Salmon dabei doch viele wichtige Probleme. So wird z. B. die Frage der deklarativen oder konstitutiven Wirkung im Grundsätzlichen vorweg beantwortet mit der geglückten knappen Definition (S. 11): „La reconnaissance est un acte par lequel un État constatant une situation de fait ou de droit admet qu'elle lui est opposable.“ 
Es folgen die erwähnte allgemeine Bibliographie und einige entsprechende Dokumente vom Protokoll von Troppau bis zur UN-Deklaration über die Prinzipien betr. freundschaftliche Beziehungen etc. zwischen Staaten von 1970. Der umfangreichere Teil des Buches enthält Materialien zu vier wichtigen Anerkennungsfällen aus der jüngeren Vergangenheit: Mandchoukouo, Katanga, Biafra und (Süd-)Rhodesien. Sie sollen zum Nachdenken und zur Diskussion anregen. Dazu gehören jeweils eine Zeittafel, relevante Dokumente, die in Thesenform zusammengefaßten Positionen der Hauptbetroffenen (also auch der UN, der OAU), ein Abschnitt mit Diskussionsthemen sowie spezielle Literaturangaben. - Es entspricht der welt- und entwicklungspolitischen Lage, daß diese Fälle in der Dritten Welt spielen. Den Nutzen aus diesem Buche wird man überall ziehen können.

\section{BRuno Simma}

Knud Krakau

\section{Das Reziprozitätselement im Zustandekommen völkerrechtlicher Verträge}

Schriften zum Völkerrecht Band 23

Duncker und Humblot, Berlin/München 1972, 68,60 DM

Wie schwierig und zugleich wichtig die Problematik der Reziprozität im Zustandekommen völkerrechtlicher Verträge ist, zeigt die vorliegende Innsbrucker Habilitationsschrift deutlich auf.

Simma will mit dieser Arbeit nicht die bereits anerkannten Problemlösungen wiederholen, sondern vor allem eigenständige Ansichten und neue Lösungen entwikkeln, die zu einer umfassenden Theorie der Gegenseitigkeit als Bauprinzip des Völkerrechts führen. Hauptanliegen ist dabei die Untersuchung der Rolle der Gegenseitigkeit in ihren verschiedenen Erscheinungsformen im Prozeß des Zustandekommens völkerrechtlicher Verträge. Der Schwerpunkt liegt dabei nicht so sehr in der klassischen Völkerrechtstheorie als vielmehr in der Klärung und Darstellung der sozialpsychologischen Situationen und Interessenlagen, die durch Reziprozitätselemente gekennzeichnet sind.

Im Eingang zu seiner Analyse untersucht Simma den Begriff der Gegenseitigkeit, den er nicht als normativen Begriff, sondern als ein in Anlehnung an die Lehrmeinung entwickeltes vielseitiges Konzept ohne eigenen materiellen Aussagewert versteht. Bei seiner anschließenden Untersuchung der Rolle des soziologisch-politisch verstandenen Reziprozitätselementes im Völkervertragsrecht geht der Verfasser auf die verschiedenen Aspekte ein: Gegenseitigkeit in der Geltung völkerrechtlicher Verträge - unter Betonung der formell-strukturellen Aspekte - und Gegenseitigkeit im Inhalt und Zustandekommen der Verträge. Im abschließenden Teil seiner Arbeit untersucht Simma die Einreihung des Reziprozitätselementes in funktionell-soziologische Typologien. Der Verfasser beleuchtet das Problem nicht nur von allen Seiten, sondern berücksichtigt bei der Untersuchung auch die problematischen Gebiete des Völkerrechts, bei denen man im ersten Hinblick die Suche nach der Reziprozität als vergeblich beurteilen würde, nämlich die Gebiete der Entwicklungshilfe, Einräumung von Handelspräferenzen an Entwicklungsländer oder das Problem einer Regelung der Meeresbodennutzung außerhalb des Festlandsockels. Wenn es hier auch an einer formell-normativen Gegenseitigkeit fehlt, so stehen im Hintergrund der Verträge stets globale Reziprozitätsüberlegungen, wie Simma überzeugend darlegt. Ein solches soziologisch-politisches Reziprozitätselement läßt sich in allen völkerrechtlichen Verträgen nachweisen. 